\title{
Using digital techniques to increase the efficiency and control of orthodontic procedures: a case study
}

\author{
L. Petitpas \\ Specialist certified in Dentofacial Orthopedics, Private practice
}

\begin{abstract}
Taking impressions with trays and PVS silicone, shipping them, producing study models, and archiving models to facilitate the orthodontic practice-these long and sometimes tedious work steps have always been readily accepted by most orthodontists as unavoidable aspects of the job.

Since the introduction of high-performance intraoral scanners for taking digital impressions, these steps are no longer necessary: shipping impressions and editing physical models is eliminated in most situations.
\end{abstract}

Instead, digital impressions can be created faster by way of a three-dimensional (3D) scanner, and the resulting files can be transmitted immediately and with ease.

These files are used as a basis for the diagnosis and planning of digital orthodontic treatment (CAD) and, increasingly, to produce fixed and removable orthodontic appliances (CAD/CAM).

However, there are greater possibilities! The virtual information obtained from intraoral impressions provides us with new means of evaluating and optimizing treatment, which we shall present here.

\section{STARTING WITH FIXED ORTHODONTICS}

In November 2014, at the age 28 years, this patient visited our office with joint and muscular disorders (clicking and pain in the right temporomandibular joint). An extraoral clinical examination revealed a mandibular deviation to the right (Fig. 1).

\section{Address for correspondence:}

Laurent Petitpas

35, impasse de la Brasserie - 54700 Pont-à-Mousson

E-mail: ortho@petitpas.eu
Article received: 26-02-2016. Accepted for publication: 12-05-2016.

This is an Open Access article distributed under the terms of the Creative Commons Attribution License (http://creativecommons.org/licenses/by/4.0), which permits unrestricted use, distribution, and reproduction in any medium, provided the original work is properly cited. 
During the intraoral examination, additional dysmorphoses were detected.

The shape of the dental arch, the canine axes, and the Wilson curve showed asymmetries (upper right maxillary linguoversion). The maxillary incisors showed significant overcrowding. An inversion of the right molar joint was observed (Figs. 2-7). A lower midline deviation was clearly visible on the frontal face X-ray (Fig. 8). Several treatment options are proposed to such
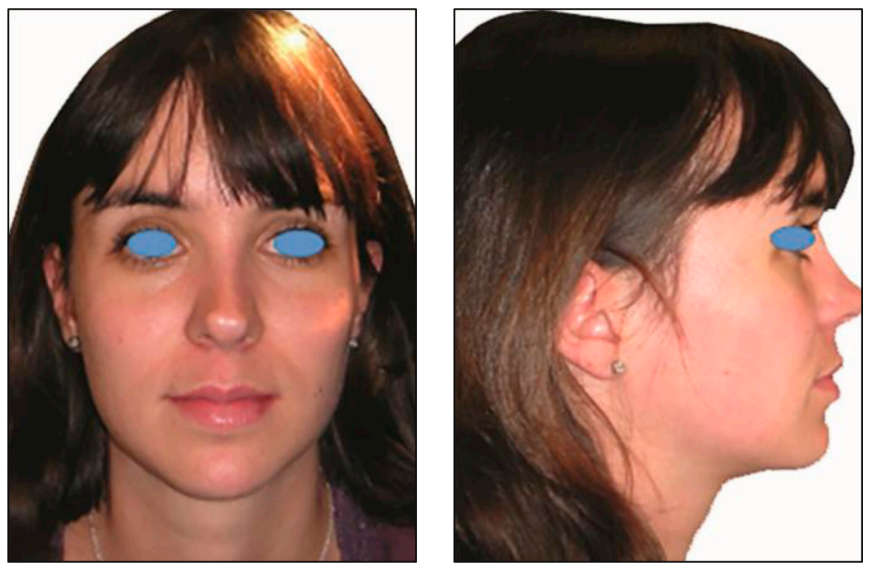

Figure 1

The esthetic examination confirmed a right mandibular deviation.

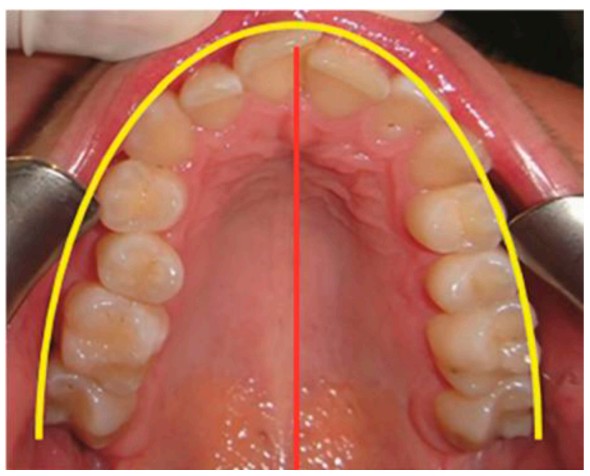

Figure 2

Asymmetric shape of the maxillary arch in comparison with the median sagittal axis visualized by the median raphe.

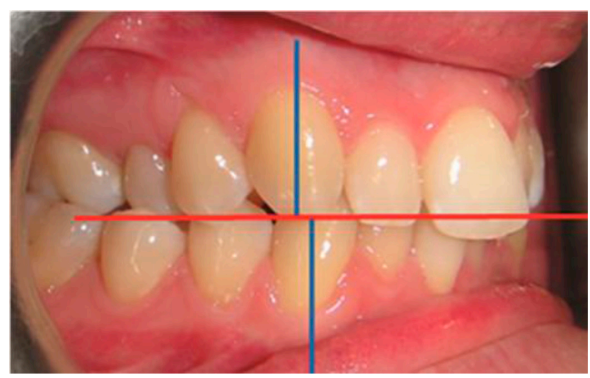

Figure 3

Class-III occlusion on the right and an inverted posterior joint.

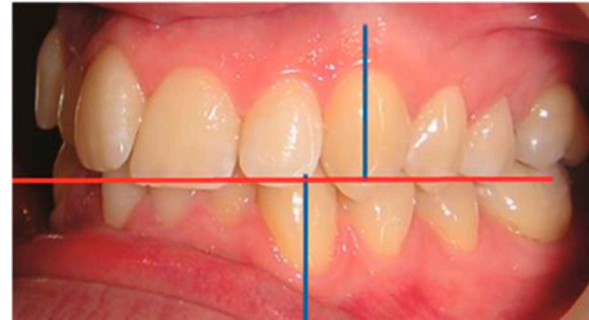

Figure 4

Class-I occlusion on the left.

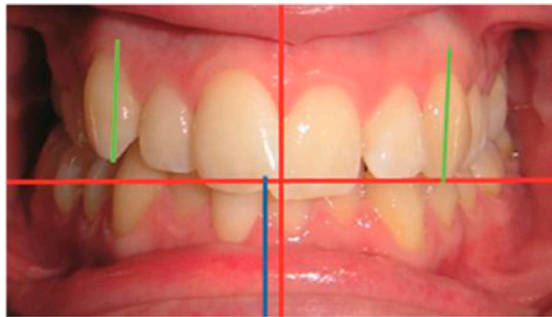

Figure 5

Analysis of the median incisors seen from the front with all dental axes shifted to the right side.

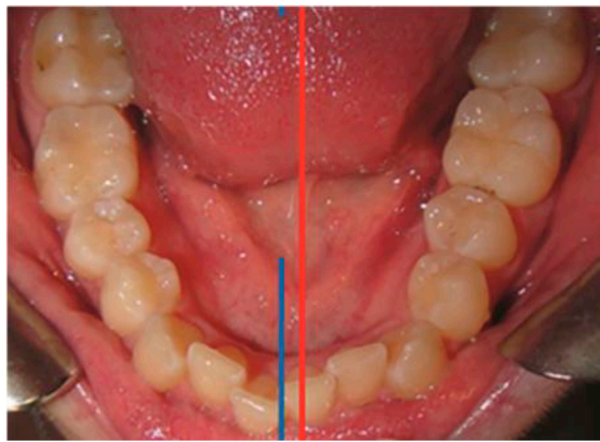

Figure 6

The median sagittal axis was deferred in relation to the lower middle incisor. 
patients who opt for an individualized lingual treatment on the maxilla.

On the mandible, a fixed vestibular treatment was planned.

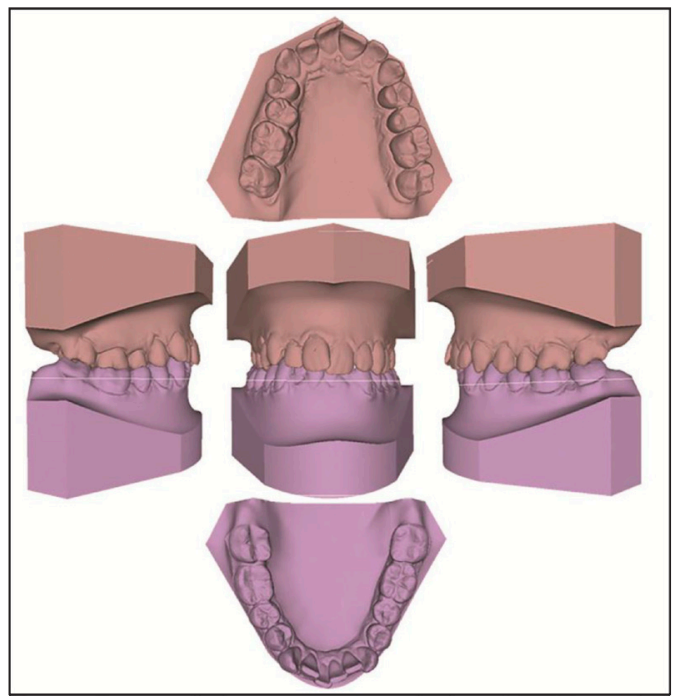

Figure 7

All these dysmorphoses can also be analyzed on exported digital casts (STL files) or in real time on the touch screen of the 3MTM intraoral True Definition Scanner.

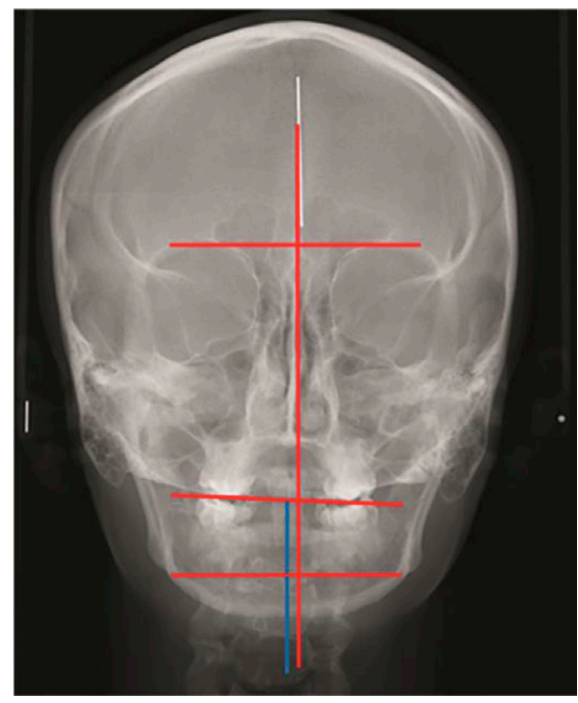

Figure 8

A frontal $X$-ray shows the deviation from the mandible against the median sagittal axis. Occlusal plane tilt.
On the virtual 3D cast, the dental arches and arch shape was simulated to visualize the asymmetries of the axes and arches (Figs. 9, 10).

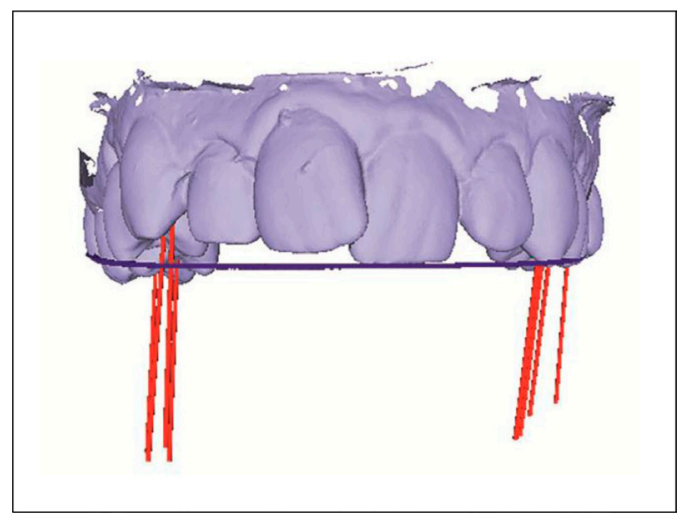

Figure 9

The dental axes can be traced to visualize the asymmetries.

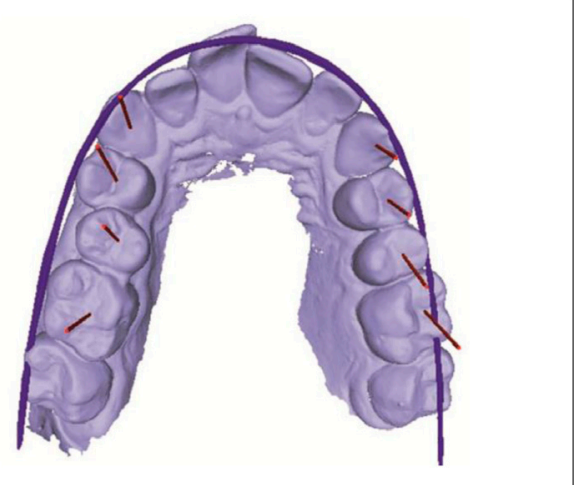

Figure 10

The dental axes and arch were plotted to view the asymmetries. 


\section{ONLINE PRESCRIPTION}

This was done after the digitization of the patient's arches and establishing the diagnosis.

Because the patient chose lingual maxillary treatment, we used the Unitek ${ }^{\mathrm{TM}}$ software; Treatment Management Portal (TMP), for Incognito 3M.

Thus, we filled out an online order form on this software. This prescription allowed users to inform the laboratory about the condition of their teeth, the desired median positions, the shape and width of the dental arch, etc (Fig. 11). The values and ratios of the molars and canines were specified. In addition, the different types of brackets, the auxiliary parts (in this case Ricketts scissors for ATP), and the arcs were selected for the maxillary device (Fig. 12).

This "online" procedure was carried out by filling out some forms on the screen, and it takes only a few minutes for someone who is familiar with it.

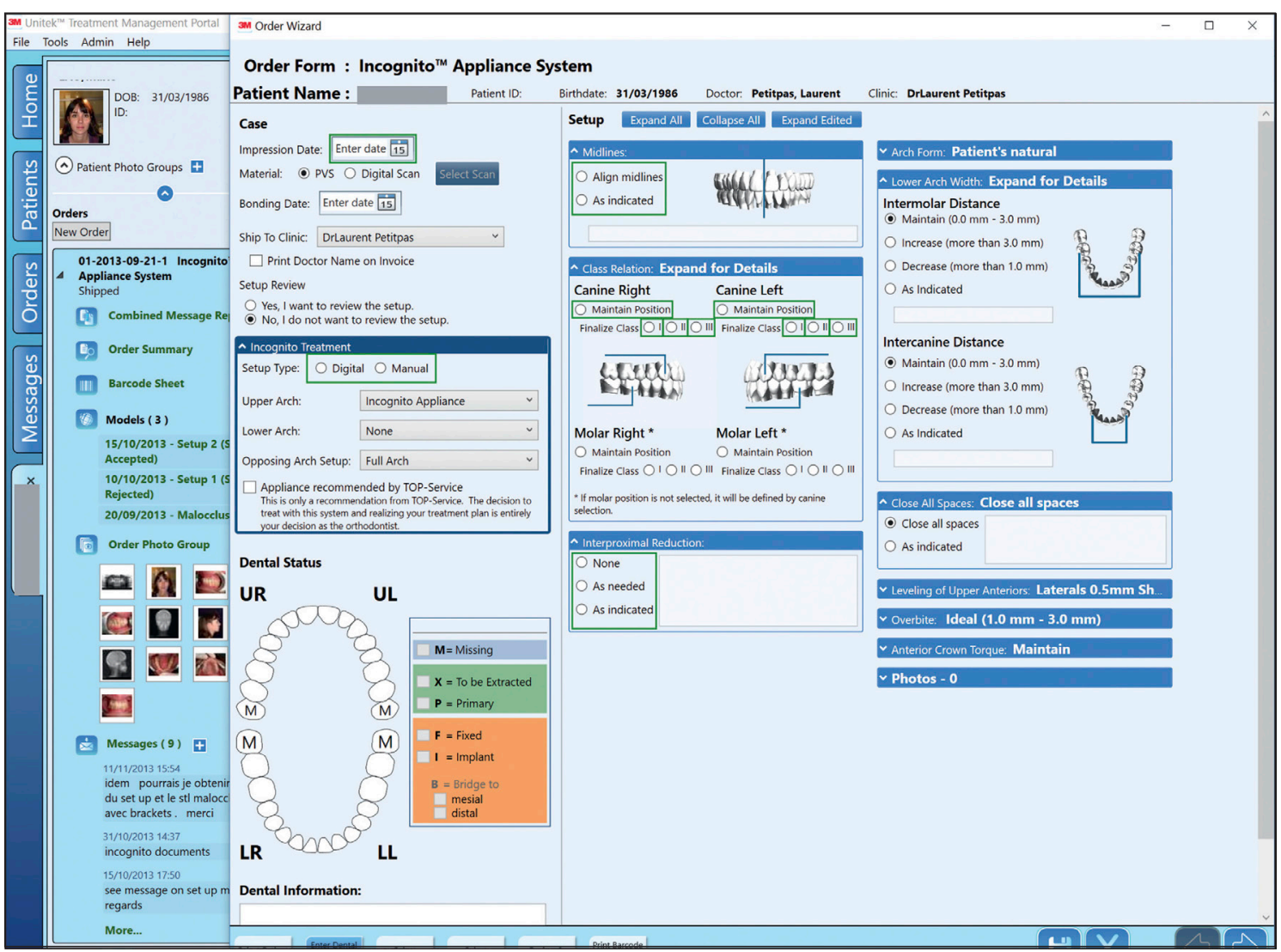

Figure 11

Treatment plan prescription and order of individualized locks on TMP. 


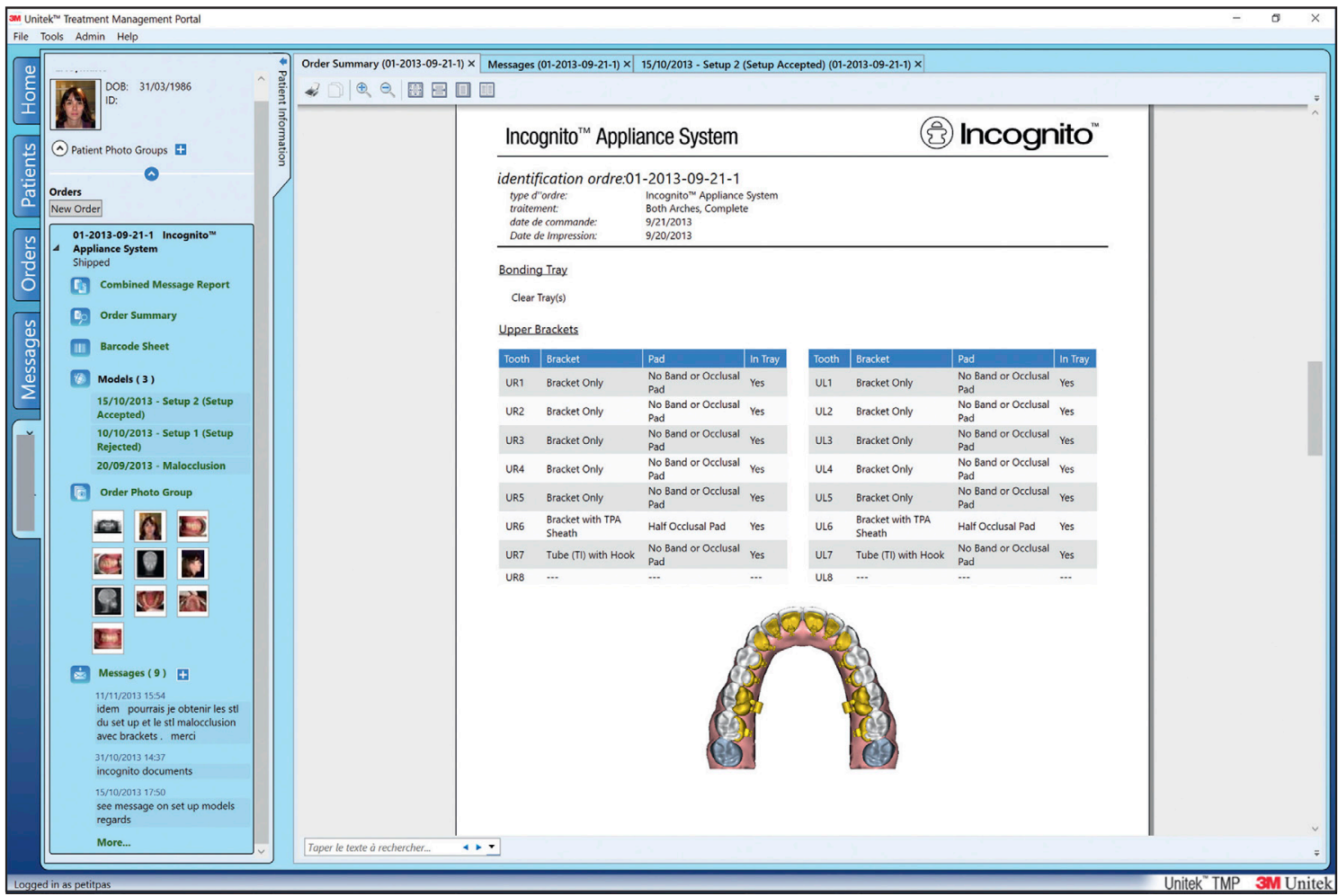

Figure 12

Order summary for brackets and locks to be used on the maxillary arch.

\section{SETUP AND VERIFICATION}

Before digital recording of the arches, the conventional plaster models of malocclusion were produced from VPS impressions, and they were digitized with an industrial scanner.

Today, these steps are eliminated with the introduction of intraoral digital impressions.

Th digital files are directly downloaded to TMP. With the treatment plan ordered online by the orthodontist, these files are simply imported into the digital setup software "SetUp Lab" (DSL).

The setup can be ordered online for both arches, even if the mandibular arch is planned to be treated via a conventional vestibular technique, as was the case in our patient. The technician creates this digital setup and uses TMP to send it to the practitioner for verification.

Figures 13 and 14 show that it is possible to view the models of occlusion and setup separately or together in the "overview" mode (Figs. 13, 14).

In addition, these models can be manipulated in all the spatial planes and measurements can be made.

For example, intermolar and intercanine distances can be measured and compared for tracking displacements. Communication is facilitated by the notes, which allow laboratory technicians and orthodontists to place annotations at selected reference points if and when corrections are necessary. 


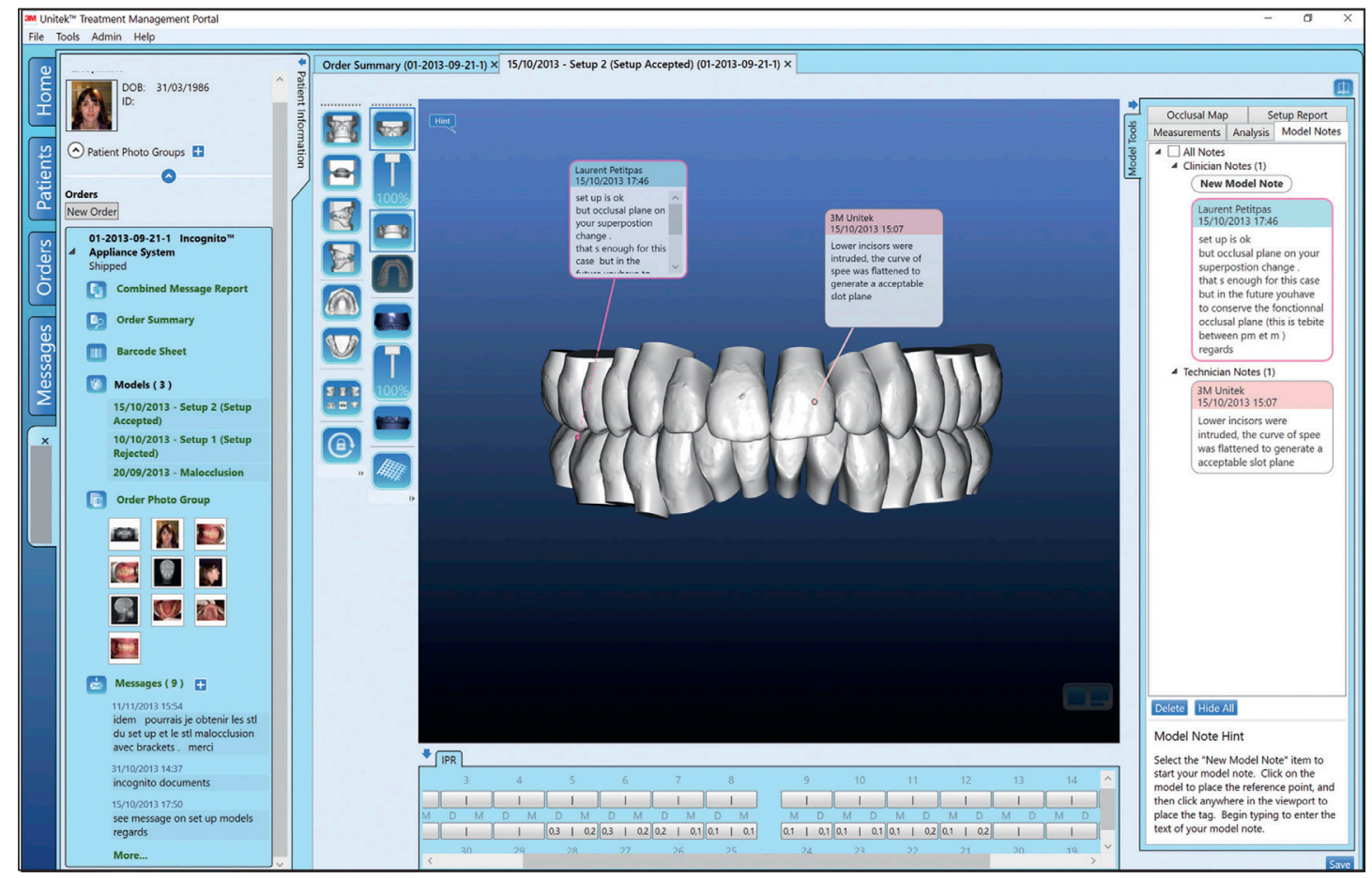

Figure 13

Setup with notes made by the technicians in the $3 \mathrm{M}$ lab.

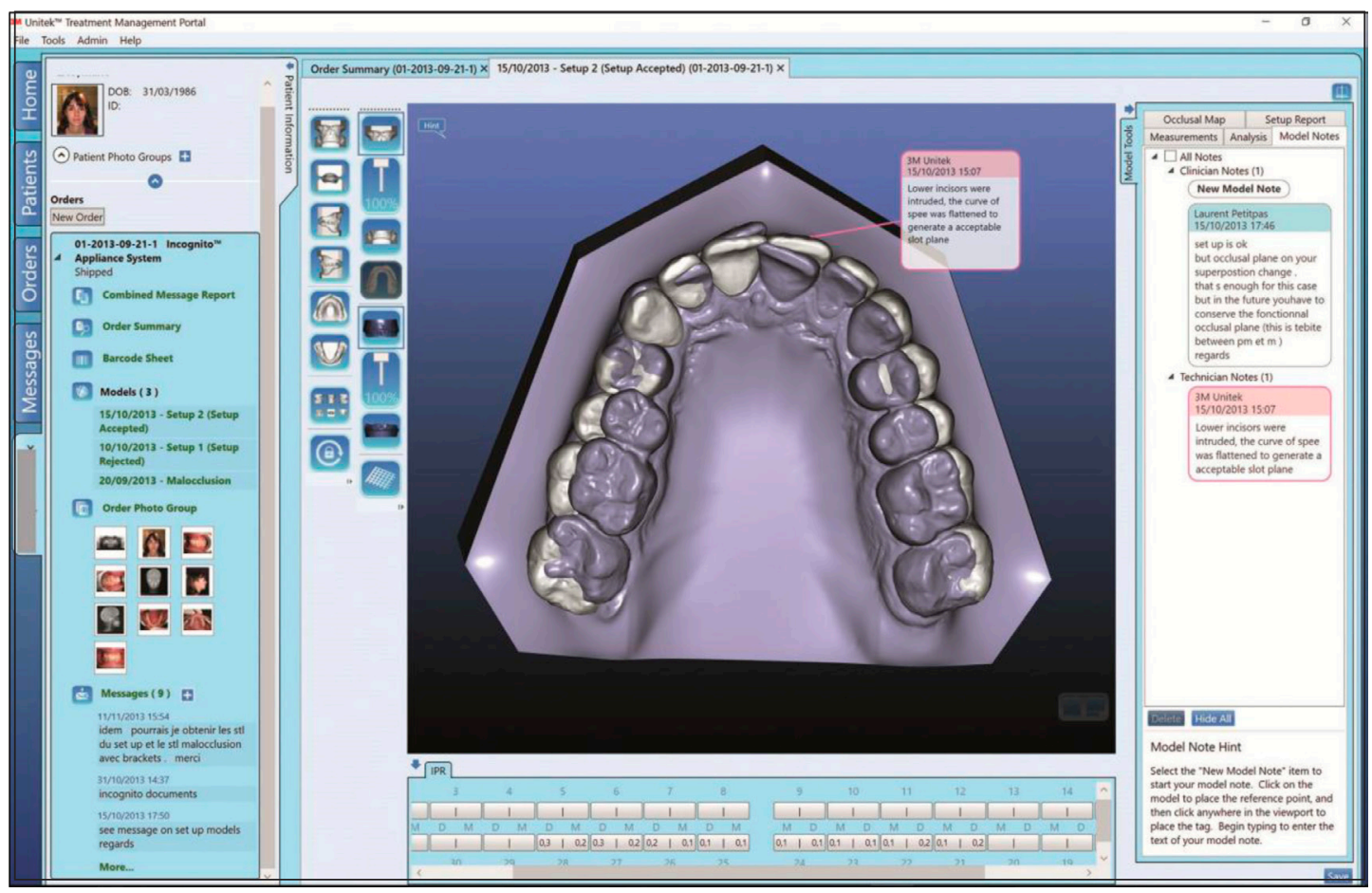

Figure 14

Setup verification in overlay on the malocclusion model in the TMP software. 


\section{INDIVIDUALIZED PRODUCTION OF THE MEDICAL DEVICE AND PLACEMENT IN THE MOUTH}

After the setup was approved, the virtual malocclusion files and setup templates were exported from TMP to check the treatment plan once again (Fig. 15). Alternatively, they could be simply stored digitally in the patient's medical record and transmitted to the office for further consultation "offline."

The brackets and arcs of the lingual system were produced by a digital process and arrived at the orthodontist's office in the form of a transparent, indirectly bonded splint. This splint was also manufactured by a digital process

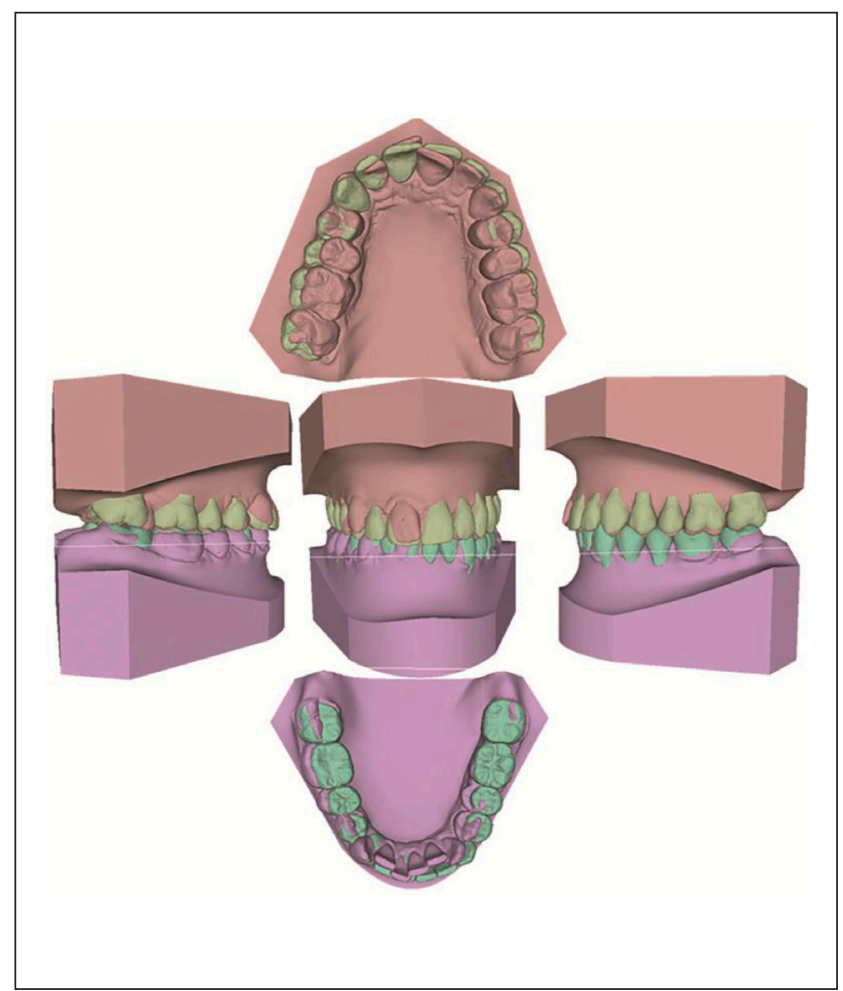

Figure 15

Malocclusion and setup files are exported in STL format.
(Incognito Clear Precision Tray ${ }^{\mathrm{TM}}$ ) and was used for precise positioning of the attachments.

Figure 16 shows the splint bonded to the maxilla, placed on a model created by 3D impression using digitized data (the impression of the model is not indispensable; Fig. 16). The brackets are bonded with resin 3M ESPE RelyX Unicem 2 Automix ${ }^{\mathrm{TM}}$ and a self-adhesive cement (Fig. 17). In the same session, the vestibular device is placed on the mandible (Fig. 18, 19).

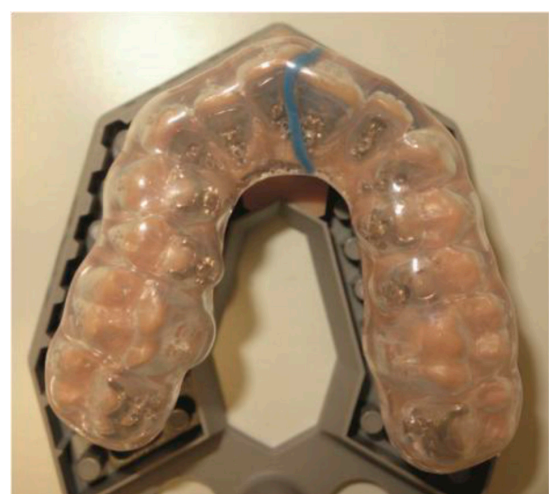

Figure 16 Incognito ${ }^{T M}$ Clear Precision Tray on a printed 3D model.

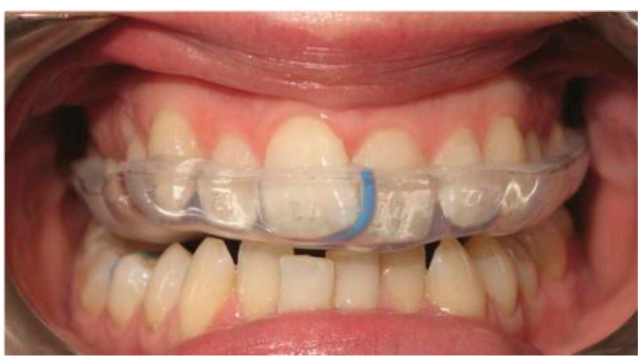

Figure 17

Indirectly bonded splint in mouth. 


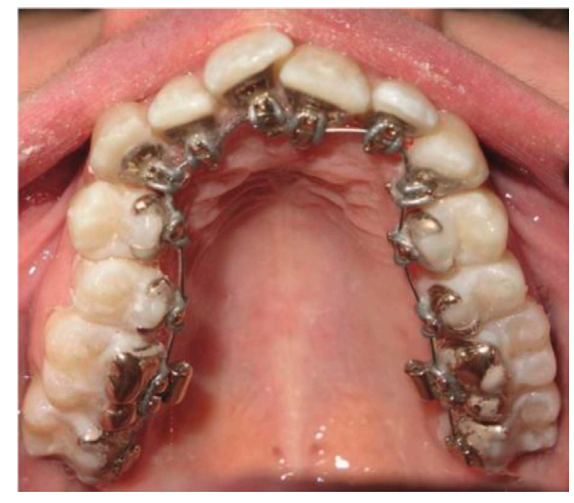

Figure 18

Situation at the beginning of maxillary arch treatment.

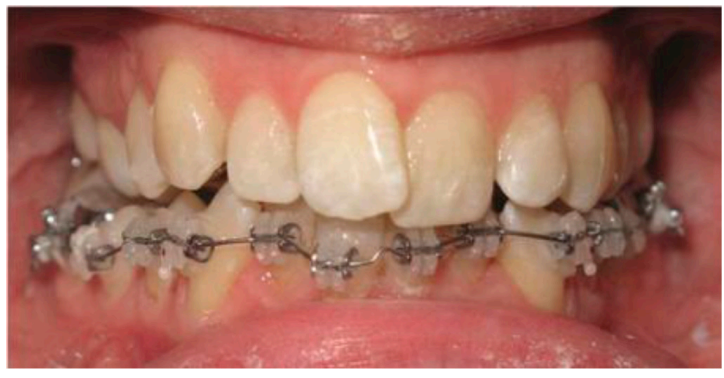

Figure 19

Anterior intraoral view with maxillary lingual and mandibular vestibular devices.

\section{PROGRESSION OF TREATMENT}

The arches had changed after 4 months and a transpalatal arch was placed in right unilateral expansion to correct arch asymmetries (Fig. 20).

An Angle's class-II malocclusion appeared after leveling, and mandibular

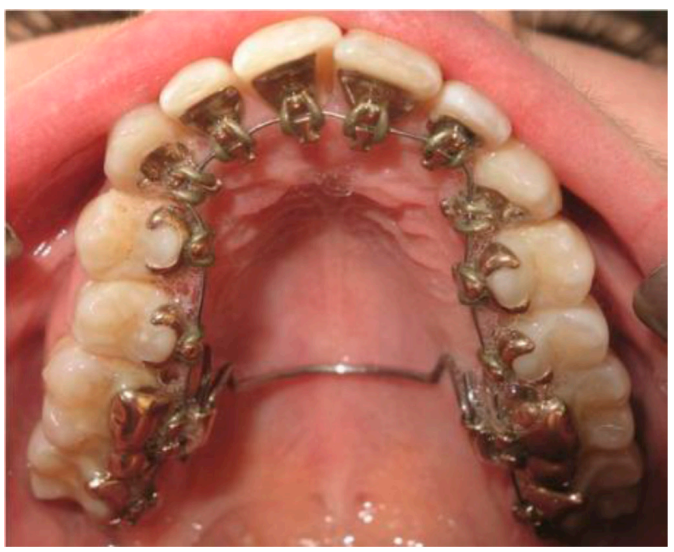

Figure 20

Transpalatal arch in right asymmetrical expansion. repositioning was needed to correct the initial deviation. At this stage, we placed occlusal wedges to facilitate articular orthopedic centering, after which we prescribed intermaxillary tractions for the patient (Fig. 21).

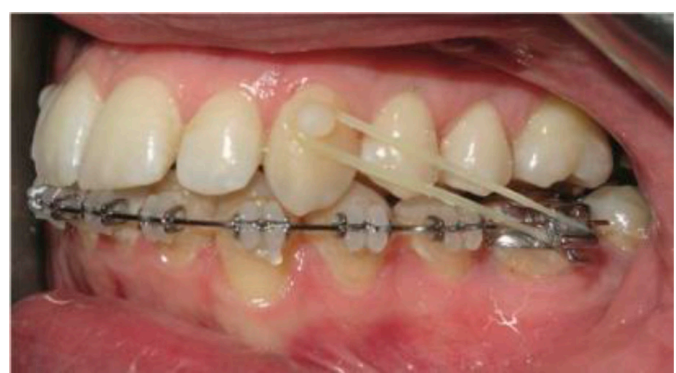

Figure 21

Correction of residual class I/ after leveling by intermaxillary traction. 


\section{FINISHING AND ACCURACY FACILITATED BY DIGITAL SYSTEMS}

When the multiattachment device was removed after 14 months of treatment (Figs. 22, 23), intraoral digital impressions were repeated over a few minutes with the patient seated in the chair.

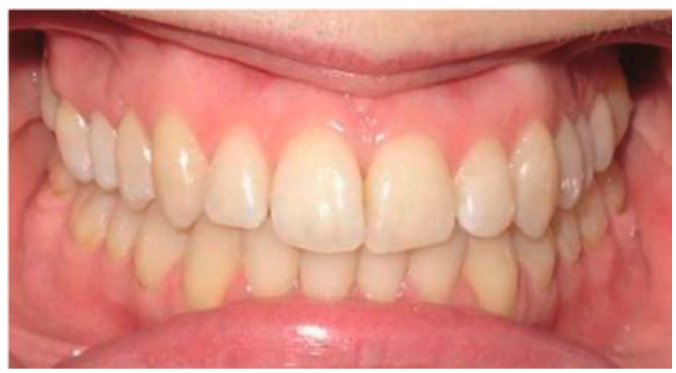

Figure 22

Result after the removal of multiband devices.
These new impressions were also downloaded in STL format.

On the one hand, these models serve as a complement of the clinical photographs, allowing the verification of the new positions of the middle incisors, jaw asymmetries, etc. (Fig. 24). On the other hand, these virtual models can be used to analyze the results obtained in comparison to the previously downloaded models at the end of treatment.

These models were thus superimposed with those of the malocclusion, the setup, and the results, which is impossible to accomplish with physical models.

This superimposition facilitates a precise and reliable comparison by superimposing on the medial palatal
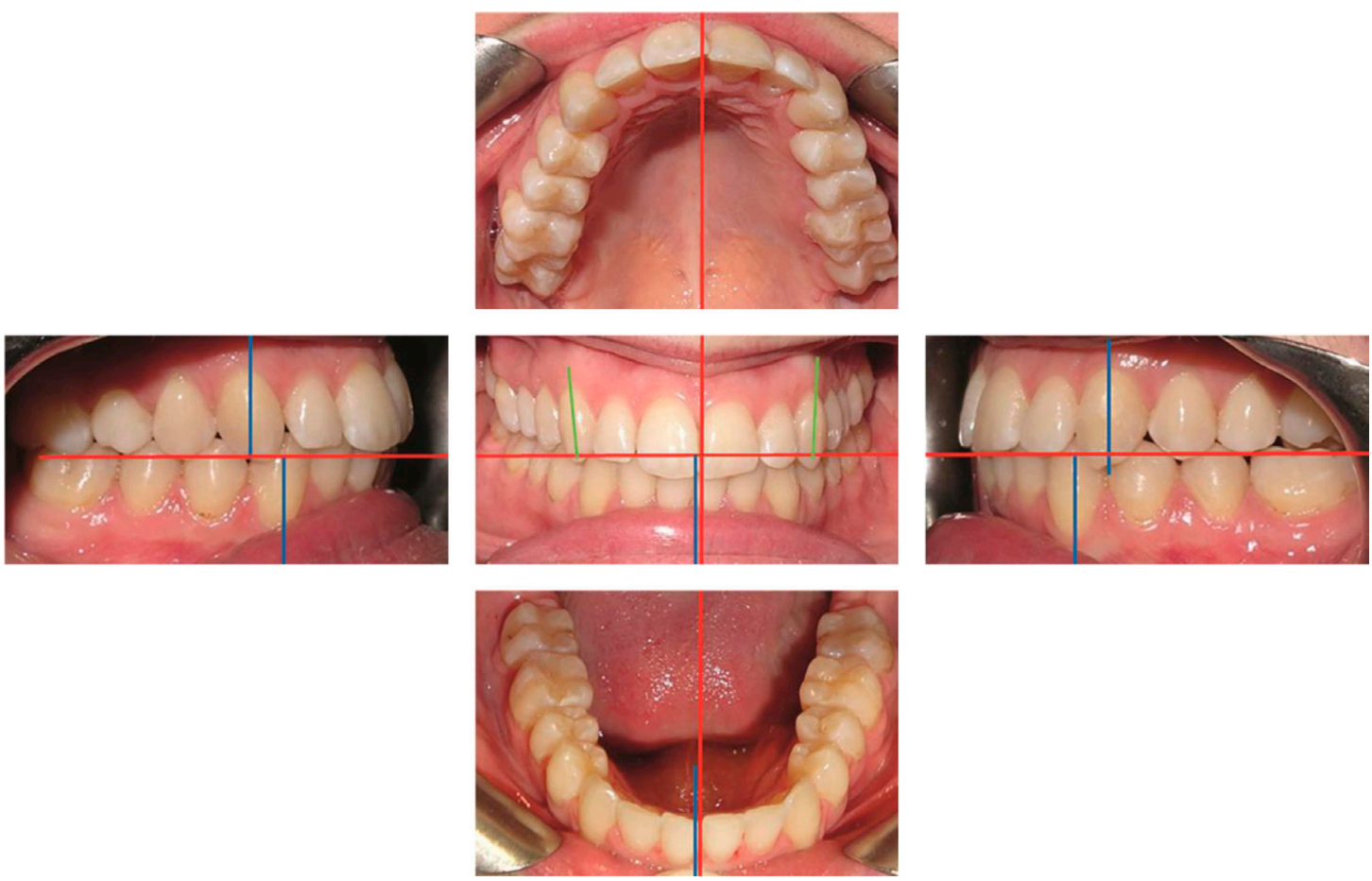

Figure 23

Evaluation of the results obtained: alignment of the midlines and horizontal occlusal planes, symmetrical canine axes, and correction of the right joint inversion. Class-l symmetrical canines and molars. 


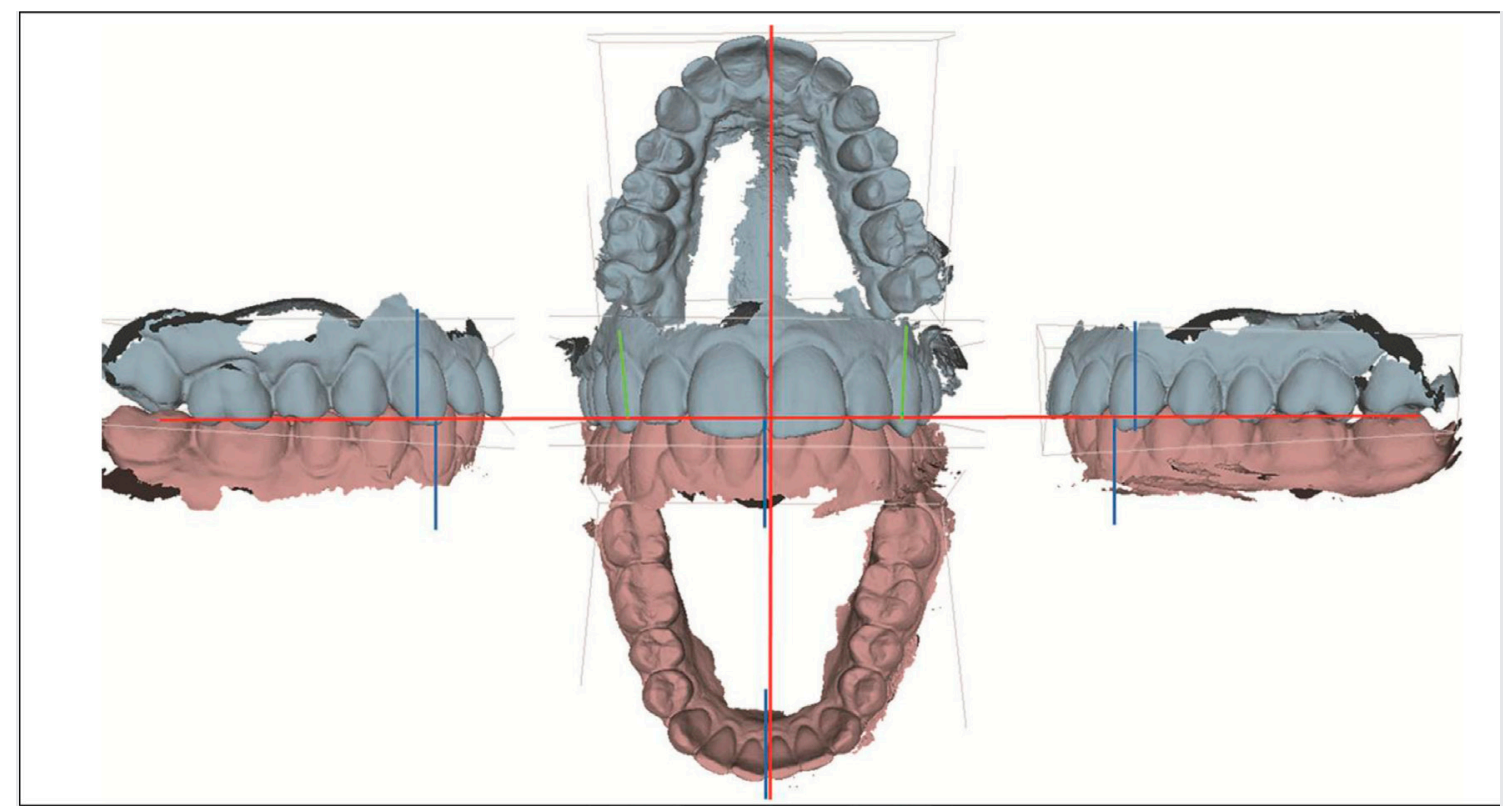

Figure 24

Verification of the results on the digital models.

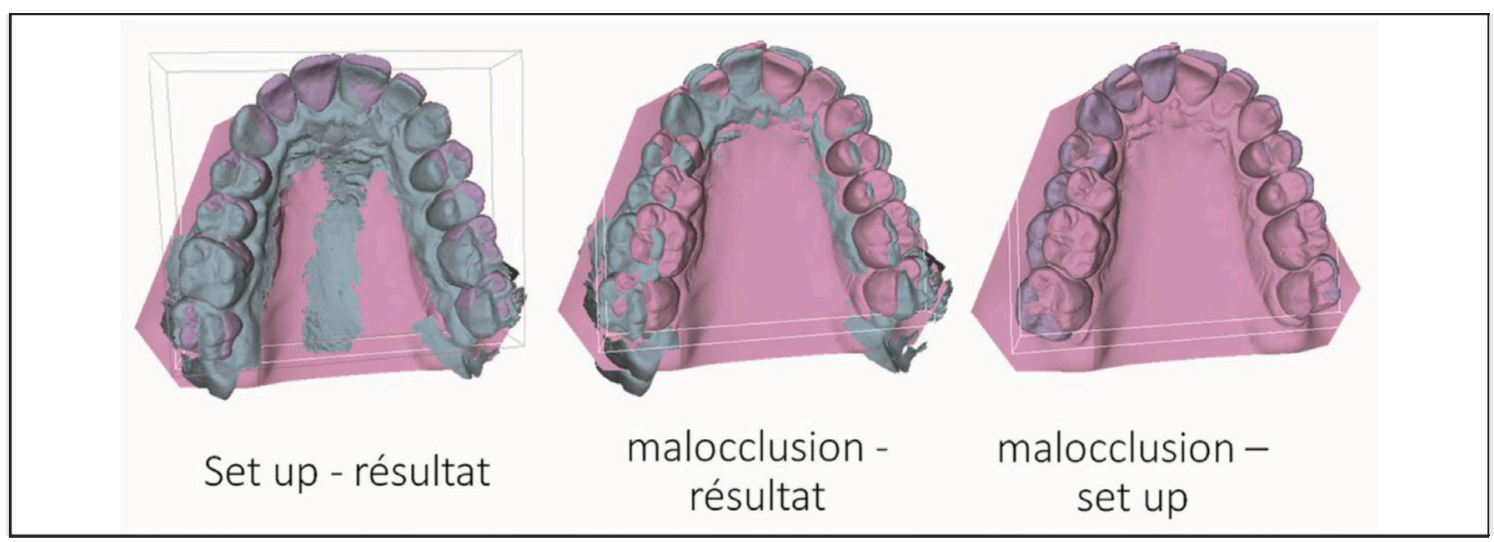

Figure 25

Digital superimposed models of initial malocclusion; setup and results of orthodontic treatment in the maxilla in the occlusal view. These images show the correction of straight unilateral asymmetries. In the maxilla, the superimposed models of the setup and the results correspond fairly accurately, which confirms the efficiency of the proposed treatment plan.

structures that were recorded during the intraoral digital impressions.

The visualization of the different possibilities of superimposing the digital models of the initial malocclusion, prediction by setup, and treatment results can all be analyzed in 3D (Figs. 25, 26).
These superimpositions show that the asymmetries have been removed as foreseen by the treatment plan developed on the basis of the setup models.

The frontal control radiograph confirmed the correction of the asymmetry (Fig. 27). 
In addition, clinical observations of the joints showed that the pains and dyskinesia of the TMJ had disappeared.

After this treatment period of 14 months with a fixed device, these final digital devices was used as a basis

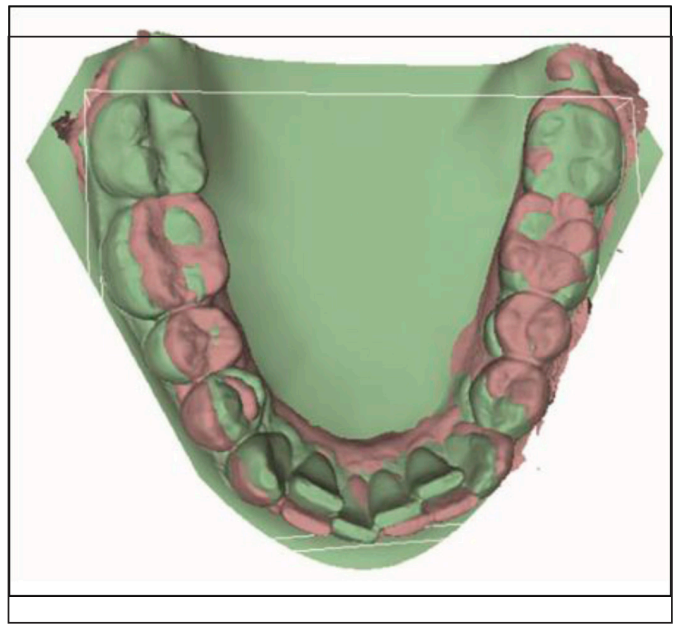

Figure 26

Superimposition between the malocclusion and the result at the mandible.

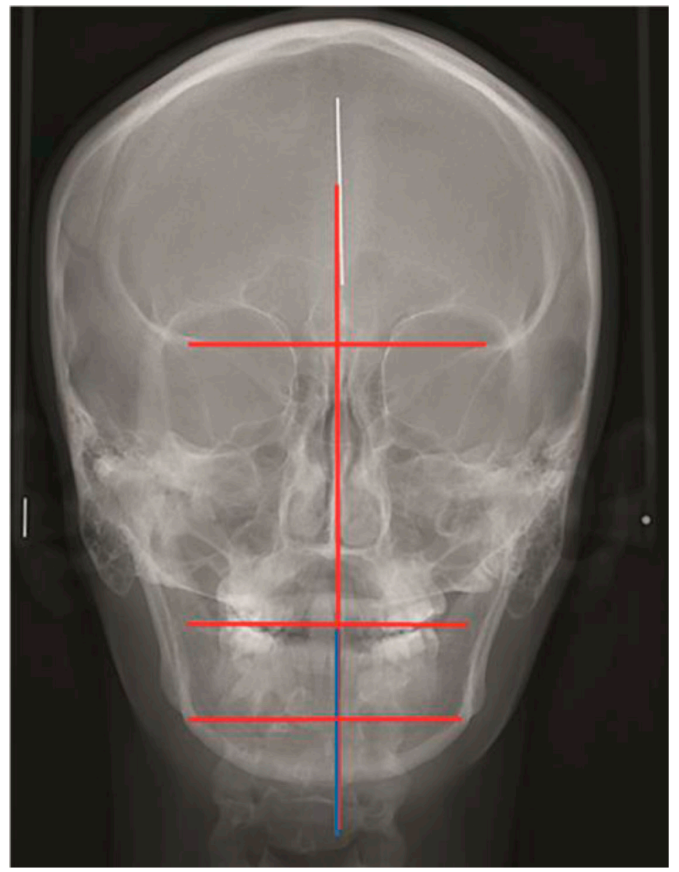

Figure 27

Face final $X$-ray shows alignment of the incisal and skeletal midlines. for planning the finishing steps with removable aligners and retainers.

A finishing setup (Fig. 28) was then developed, and physical models were edited with a 3D printer at the orthodontists' office. These printed models were

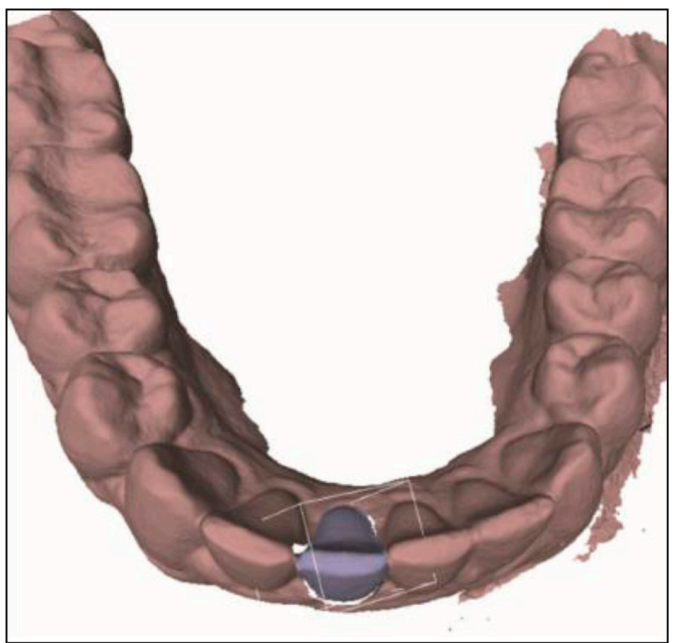

Figure 28

Setup of the final correction of the finishing treatment.

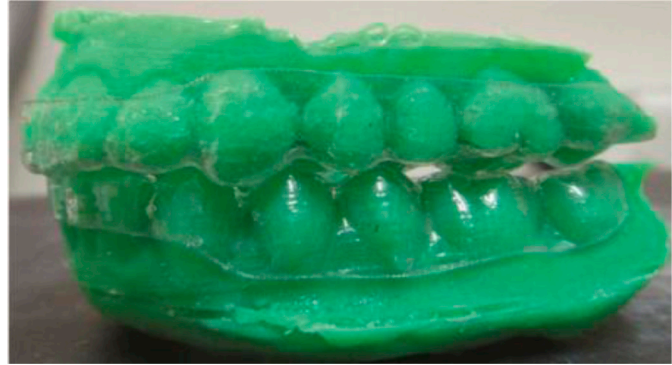

Figure 29

Thermoformed aligners produced on a printed 3D model. 

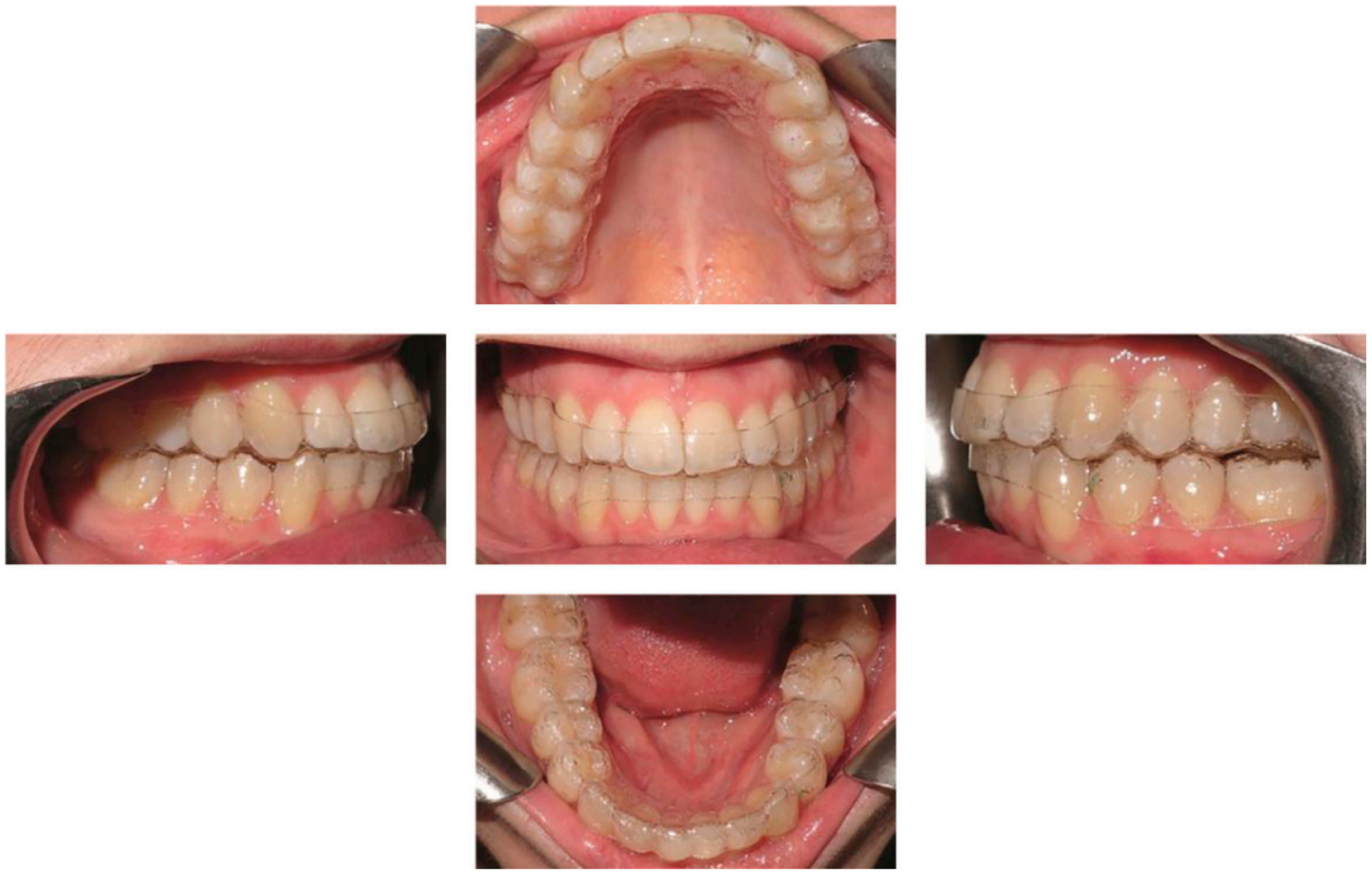

Figure 30

Finishing aligners in the mouth.
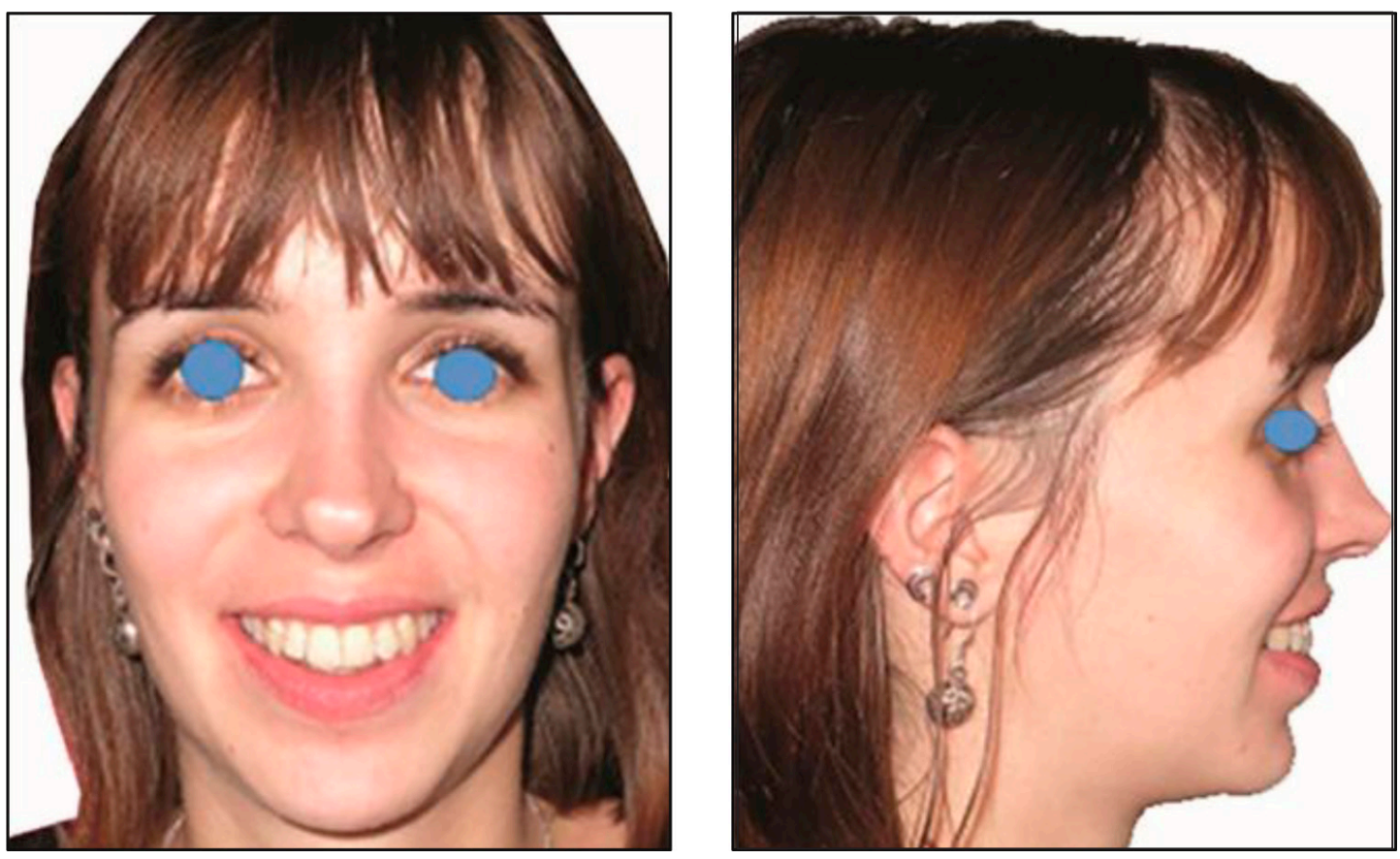

Figure 31

Extraoral photo of the smile with aligners placed. 
then used to produce aligners by thermoforming (Fig. 29). These splints made it

\section{CONCLUSION}

As shown in this presentation, intraoral digital impressions are able to not only replace PVS in the treatment procedures but also assist orthodontists to improve the diagnosis and treatment as well as to better control the outcomes. The digital data produced by 3D intraoral scanning can be possible to perfect the multiband orthodontic treatment (Figs. 30, 31).

used and compared for better control over the movements of the teeth. Consequently, this allows us to develop a more precise understanding of orthodontic processes.

Conflict of interest: The author declares that they do not have any conflict of interest. 